\title{
Fabrication and Characterization of Heparin/Collagen Sponge for in Vitro Differentiation of Wharton's Jelly-Derived Mesenchymal Stem Cells into Hepatocytes
}

\author{
Fatemeh Aleahmad, ${ }^{1}$ Tahereh Talaei-Khozani, ${ }^{2}$ Sareh Rajabi-Zeleti, ${ }^{3}$ Mahsa Sani, ${ }^{2}$ Sasan \\ Jalili-Firoozinezhad, ${ }^{3,4}$ Shahin Bonakdar, ${ }^{5}$ Sanaz Heshmat-Azad, ${ }^{6}$ Mahnaz Azarnia, ${ }^{1,{ }^{*}}$ and Mansoureh \\ Jaberipour $^{7}$ \\ ${ }^{1}$ Department of Animal Biology, Faculty of Biological Sciences, Kharazmi University, Tehran, IR Iran \\ ${ }^{2}$ Tissue Engineering Lab, Shiraz Medical School, Shiraz University of Medical Sciences, Shiraz, IR Iran \\ ${ }^{3}$ Department of Stem Cells and Developmental Biology at Cell Science Research Center, Royan Institute for Stem Cell Biology and Technology, ACECR, Tehran, IR Iran \\ ${ }^{4}$ Departments of Biomedicine and Surgery, University Hospital Basel, University of Basel, Hebelstrasse, Basel, Switzerland \\ ${ }^{5}$ National Cell Bank of Iran, Pasteur Institute of Iran, Tehran, IR Iran \\ ${ }^{6}$ Department of Chemistry, Isfahan University of Technology, Isfahan, IR Iran \\ ${ }^{7}$ Institute of Cancer Research Center, Shiraz University of Medical Sciences, Shiraz, IR Iran
}

"Corresponding author: Mahnaz Azarnia, Department of Animal Biology, Faculty of Biological Sciences, Kharazmi University, Tehran, IR Iran. P.O. Box: 15719-14911. Tel: +98-2188848940, Fax: +98-2634510004, E-mail: elaheh_aleahmad@yahoo.com

Received 2016 July 12; Revised 2016 October 16; Accepted 2017 January 01.

\begin{abstract}
Background: Recapitulating the native cell niche and extracellular matrix (ECM) architecture in vitro helps reconstruct injured tissues. Collagen I and heparin are two important constituents of the ECMs, which play crucial roles in regulating cell behaviors. Specifically in the liver, these components can affect the differentiation and functionality of the cells.

Objectives: The aims of this study were to first fabricate and characterize a heparin/collagen scaffold and then investigate the scaffold's efficiency in directing the differentiation of Wharton's jelly-derived mesenchymal stem cells (WJ-MSCs) towards hepatocyte. Methods: After fabricating the rat tail collagen I sponge-shaped scaffolds, heparin was chemically immobilized on the scaffolds using N-(3-dimethylaminopropyl)-N-ethylcarbodiimide hydrochloride (EDC) and N-hydroxysuccinimide (NHS). The scaffold chemical characteristics and architecture were evaluated by Fourier-transformed infrared (FTIR) spectroscopy and scanning electron microscopy (SEM), respectively. In the next step, Wharton's jelly-derived mesenchymal stem cells were seeded on the scaffolds and cell viability and morphology were assessed using MTT assay and SEM, respectively. Moreover, followed by exposing the WJ-MSCs to the hepatogenic media for 3 weeks, liver-specific marker expression and indocyanine green (ICG) clearance tests were performed.

Results: The data showed that $0.25 \mathrm{mg} / \mathrm{mL}$ heparin had no detrimental effects on the cell viability and proliferation compared to the observed effects in non-heparinized conditions. SEM micrographs showed that while immobilizing heparin had no considerable effect on the porosity of the scaffolds, the mean value of the pore sizes of heparinized sponges was higher than that of non-heparinized ones. The hepatocyte differentiation appeared to be enhanced in the cells cultured in heparinized sponges as indicated by higher percentage of the cells expressing cytokeratin 19 and albumin as well as improved indocyanine green clearance levels.

Conclusions: Heparin/collagen scaffold serves as a good platform for promoting differentiation into hepatocytes and therefore, it has the potential to be considered for drug discovery and liver regenerative medicine.
\end{abstract}

Keywords: Wharton's Jelly-Derived Mesenchymal Stem Cells, Hepatocytes, Collagen, Heparin, Tissue Engineering, Scaffold

\section{Background}

According to the world health organization reports, $2.5 \%$ of the total deaths all around the world are due to liver diseases (1), and these conditions are projected to become the 14th most common cause of death by 2030 (2). Although liver shows a good regeneration potential, drugs, toxins or viral infections have harmful effects on the hepatocytes; thus, they reduce the liver's functionality and regeneration capacity. Recently many studies have fo- cused on optimizing the scaffolds used in tissue engineering prospects. The combination of stem cell therapy and tissue engineering is a new promising approach.

Different materials have been proposed as the components of scaffolds used in liver tissue engineering applications. Natural-based substrates have attracted increasing attention due to their excellent biocompatibility and outstanding potential to provide bioactive cues (3). Collagen is one of the natural biomaterials that have been used in different forms such as collagen gels (4), sand- 
wiches (5), microspheres (6), and coatings (7), to culture primary hepatocyte. This substance has many cell-binding motifs, low antigenicity, and high biocompatibility and biodegradability. While hepatocytes lose their functions (8) and proper morphology (9) after isolating and during the conventional culture procedures, collagen-based scaffolds can boost their function (10). Since collagen is one of the most important components of the liver ECM and given the fact that culturing the hepatocytes on the scaffolds containing liver ECM results in up-regulation of liver-specific genes (11), collagen-based materials have been shown to improve the differentiation of the stem cells toward hepatocytes (12).

Heparin, as an anticoagulant agent, has a pivotal role in angiogenesis and reducing inflammation (13). The main feature of heparin which makes it an appropriate agent for tissue engineering applications lies in its high affinity to growth factors such as fibroblast growth factors, hepatocyte growth factor (HGF), platelet-derived growth factor, vascular endothelial growth factor (VEGF), and bone morphogenic protein-6. This affinity facilitates the growth factor binding and stabilizing and avoids thermal degradations (14). Heparin, a naturally occurring glycosaminoglycan (GAG), is found in the Disse space of the liver and can form a complex with HGF (15), a known growth factor for hepatocytes differentiation. Heparin-hydrogel has been shown to improve the hepatocyte functions and viability (16). The advantage of these constructs is twofold: first, due to providing the mentioned bioactivity, the cells produce a higher amount of albumin and hence, hepatocyte phenotype is maintained in these constructs (17). Second, they provide a three-dimensional (3D) environment to cultivate the cells. 3D culture condition, for instance, has been shown to modify the liver-specific gene expression pattern in MSCs (18). Also, an improvement in hepatocyte function and bio-mimicking of toxicity response has been reported through 3D culture (19). The 3D culture condition can lead to the acceleration of hepatocyte maturation (20). GAGcontaining collagen scaffolds have been previously used in tissue engineering context for both in vivo and in vitro studies to improve cellular attachment, metabolism, viability, as well as cell-matrix interactions (18). Cell attachment, cell-cell interactions, and gene expression are also influenced by culture conditions.

\section{Objectives}

The aim of the present study was to evaluate the synergistic effect of biological cues and microstructure environment of collagen and heparin. Although heparinized scaffolds have been previously studied in cartilage and bone tissue engineering, this study, to the best of our knowledge, for the first time shows this scaffold can find utilization for liver tissue engineering approaches.

\section{Methods}

\subsection{Scaffold Fabrication}

To obtain the collagen scaffolds, collagen type I was extracted from rat tail followed by treatment with acetic acid as previously reported (21). To generate the scaffolds, collagen solution was initially lyophilized by freeze dryer (Christ, Alpha 1-2 LD, Germany) overnight to obtain collagen powder. Next, $10 \mathrm{mg} / \mathrm{mL}$ of collagen powder was dissolved in $0.5 \mathrm{M}$ acetic acid $(1 \% \mathrm{w} / \mathrm{v})$ on ice and $1 \mathrm{~mL}$ of the mixture was poured into a well of a 24 -well plate culture dish (Jetbiofil, China). The plate was kept at $-70^{\circ} \mathrm{C}$ overnight and subsequently freeze-dried to form a sponge-shape disc (22).

Collagen sponges were then chemically crosslinked for 24 hours at $4^{\circ} \mathrm{C}$ within a sterile solution of 50 $\mathrm{mM}$ 2-N-morphlino ethanesulfonic (MES, Sigma, USA) in $70 \%$ ethanol ( $\mathrm{pH}$ 5.4) containing 5mM1-ethyl-3-[3dimethylaminopropyl] carbodiimide (EDC, Sigma, USA) and $2 \mathrm{mM}$ of N-hydroxysulfosuccinimide (NHS, Sigma, USA). Thereafter, the serial amount of heparin sodium salt (Hepalink, China), $0.25,0.5$, or $1 \mathrm{mg}$, was added to 1 $\mathrm{mL}$ crosslink solution (EDC/NHS) and incubated for 30 minutes to activate the heparin. Next, the solution containing heparin was added to the collagen sponges for 24 hours at $4^{\circ} \mathrm{C}$. Thereafter, the scaffolds were treated with $\mathrm{Na} 2 \mathrm{HPO} 4(0.1 \mathrm{M})$ for 2 hours to inhibit the crosslinking reaction. To prepare the samples for cell culture, sponges were rinsed several times with sterile double distilled water and incubated for 24 hours in phosphate buffer saline (PBS) with refreshing the PBS every 8 hours. The crosslinked sponges were frozen at $-80^{\circ} \mathrm{C}$ and freeze-dried until further use. The sponge was then sterilized by $70 \%$ ethanol. The control sponges were prepared in the same way but without incubation with any heparin sodium. After evaluating the impact of heparin concentration on cell viability, the sponges with the most viable cells were chosen for further in vitro evaluations.

\subsection{Macroscopic Characterization of Sponges}

The presence of heparin in heparin/collagen samples was evaluated by adding $0.0005 \%$ toluidine blue in 0.001 $\mathrm{N}$ hydrochloric acid with $0.02 \%(\mathrm{w} / \mathrm{v})$ sodium chloride for 1 minutes, as previously described (23). The dye was then removed and the samples were washed with PBS several times and digital images were captured. 


\subsection{Fourier Transform Infrared (FTIR) Spectroscopy}

Infrared spectra of heparinized and non-heparinized collagen sponge were obtained using a Bruker FTIR spectrometer (Vertex70, Germany). Absorbance spectra were recorded at a resolution of $8 \mathrm{~cm}^{-1}$ in the range of 4000 to $400 \mathrm{~cm}^{-1}$. The spectra were obtained from a set of four samples, namely non-crosslinked collagen, crosslinked heparinized, non-heparinized collagen, and heparin sodium. All samples were dried in freeze-dryer and minced into very small pieces and mixed with potassium bromide before analysis.

\subsection{Wharton's Jelly-Derived Mesenchymal Stem Cells Isolation and Culture}

Wharton's jelly-derived mesenchymal stem cells (WJMSC) were isolated, as reported elsewhere (24), from the umbilical cords obtained from the infants delivered by cesarean section while informed consent was obtained from their parents. The umbilical cord vein was flashed with PBS, the arteries were removed, and the rest were cut into small pieces. The pieces were plated on culture flask in the presence of $\alpha$-minimal essential medium ( $\alpha$ MEM, Bioidea, Iran) containing $10 \%$ fetal bovine serum (FBS, Gibco, UK), 1\% L-glutamine (Bioidea, Iran), and 1\% penicillin/streptomycin (Gibco, UK). The cells at passage 3 were used for this study.

\subsection{Wharton's Jelly-Derived Mesenchymal Stem Cells Character- ization}

To characterize WJ-MSCs, an aliquot of $1 \times 10^{6} \mathrm{cell} / \mathrm{mL}$ was washed with PBS and non-specific binding sites were blocked by incubating the cells with PBS containing 10\% FBS. The cells were then incubated in FITC-conjugated antiCD44 and CD144, phycoerythrin-conjugated anti-CD34 and CD106, and PerCP-conjugated anti-CD105 antibodies (all from Abcam, UK) for 30 minutes. The frequency of the positive cells was assessed by FACS calibrated instrument (BD, USA). To evaluate the pluripotency of the WJ-MSCs, the cells were exposed to osteogenic (MACS, Germany) and adipogenic media (StemCell Technologies Inc, Canada) for 4 and 3 weeks, respectively. The osteoblast differentiation was assessed by detection of Ca precipitation using alizarin red S staining. The adipocyte differentiation was detected by the presence of lipid droplets within differentiated cells using oil red $\mathrm{O}$ staining.

\subsection{Cell Seeding on Sponges}

After sterilization, $5 \times 10^{4}$ and $4 \times 10^{5}$ cells per well of a 24-well plate were seeded on each sponge for viability assay and the cell morphology and hepatogenic cell differentiation were studied within the constructs.
3.7. Scanning Electron Microscopy (SEM) and Histological Studies

To study the ultrastructure of the heparinized scaffold and cell morphology on the scaffolds, after 7 days, cell-seeded and cell-free scaffolds were fixed with $2.5 \%$ glutaraldehyde, dehydrated in gradually decreased ethanol and Hexamethyldisilizane (Merck, USA), and air-dried. The scaffolds were first coated with gold using a sputter coater and subsequently assessed by SEM (KYKY/EM 3200, Japan). The fiber diameter and orientation were evaluated using the Image analyzer software (http://imagej.nih.gov/ij/index.html).

Some sponges were washed with PBS, fixed with 10\% PBS/neutral buffered formalin solution ( $\mathrm{pH} 7.4$ ), and prepared for paraffin sectioning. The paraffinized blocks were sectioned at a thickness of $5 \mu \mathrm{m}$. The sections were rehydrated in ethanol and stained with hematoxylin and eosin (H and E, Sigma) and Hoechst (Invitrogen, USA) for 3 minutes. The sections were observed with fluorescence microscopy to study the cell distribution and density.

\subsection{Cell Viability Assessment}

The cell viability and proliferation were evaluated using 3-(4, 5-dimethylthiazol-2-yl)-2, 5-diphenyltetrazolium bromide (MTT, Sigma) assay. The cells were seeded at a density of $5 \times 10^{4}$ per each well of a 24 -well plate for 3, 7, and 14 days. The culture media were then replaced by $1 \mathrm{mg} / \mathrm{mL}$ of MTT in DMEM and incubated at $37^{\circ} \mathrm{C}$, in the presence of $5 \% \mathrm{CO} 2$ in dark environment for 3 hours. The dye was then eluted by Dimethyl sulfoxide and optical density was measured at $595 \mathrm{~nm}$.

\subsection{Hepatogenic Differentiation}

To study the potential of the sponges for inducing hepatocyte differentiation, WJ-MSCs at a density of $4 \times 10^{5}$ per well of a 24-well plate were exposed to hepatogenic medium. Hepatogenic medium included Dulbecco's MEM (DMEM) containing 10\% FBS, 20 ng/mL HGF (Peprotech, UK), $20 \mathrm{ng} / \mathrm{mL}$ insulin-like growth factor 1 (IGF-1, Peprotech, UK), 100 nM dexamethasone (Sigma, USA), with 1\% Lglutamine, and 1\% penicillin/streptomycin. After 7 days, $10 \mathrm{ng} / \mathrm{mL}$ of oncostatin $\mathrm{M}$ (Peprotech, UK) was added to the hepatogenic medium to induce hepatocyte differentiation. The cells were incubated for further 14 days.

\subsection{Immunohistochemistry}

On the day 21 after induction of differentiation, the sponges were fixed in 10\% formalin for later histological assessment. To do so, the samples were cut into $5 \mu \mathrm{m} \mathrm{sec}-$ tions. The tissue slides were prepared for immunofluorescence. The non-specific binding sites were blocked with 
PBS containing 10\% goat serum and Tween 20 (Merck, USA). The samples were stained with FITC conjugated-anti albumin antibody (Abcam, UK) and primary anti-cytokeratin18, -19 and alpha-fetoprotein antibodies (Abcam, UK) at dilution of 1:100. The specimens were treated with FITCconjugated donkey anti-rabbit antibody for cytokeratin18 and -19 antibodies (Santacruz, USA) at dilution of 1: 300 and FITC conjugated anti-mouse antibody for alphafetoprotein at a dilution of 1:160 (Abcam, UK). Then, the samples were counterstained with Hoechst (Invitrogen, USA) and mounted with anti-fade medium. The corresponding FITC-conjugated isotype antibodies were used as control. The intensity of the reactions of the differentiated cells to all antibodies was evaluated using Image J software. The frequency of the positive cells for each antibody was calculated by choosing random fields and counting the stained and non-stained cell populations.

\subsection{Reverse Transcription-Polymerase Chain Reaction (RT-PCR)}

The harvested cells were resuspended in $1 \mathrm{~mL}$ Biozol (Bioflux) to detect the expression of albumin. The total RNA was extracted by chloroform/isopropanol. cDNA was synthesized by cDNA synthesis kit (Fermentas, USA). The forward and reverse primer sequences were $\mathrm{F}$ 5'ACAGAGACTCAAGTGTGCCAGT-3' and R 5'GCAAGGTCCGCCCTGTCATC-3', respectively. The PCR products were run on $2 \%$ agar gels.

\subsection{Indocyanine Green Clearance Assay}

The culture media were replaced by $1 \mathrm{mg} / \mathrm{mL}$ of indocyanine green (Sigma, USA) in DMEM for 15 minutes. Subsequently, the indocyanine green solution was removed and common culture media replaced for next 6 hours. The absorption and release of the dye were evaluated by assessing the optical density of the media at $820 \mathrm{~nm}$ (25). HepG2 was used as positive control and undifferentiated WJ-MSCs were used as negative control.

\subsection{Statistical Analysis}

All data were expressed as mean \pm standard deviation (SD). The analyses of variance (ANOVA) and LSD test were used to analyze the data. The analyses were performed by Excel 2010. P values less than 0.05 were considered as significant difference. All experiments were performed in triplicate.

\section{Results}

\subsection{Cell Characterization}

To characterize the WJ-MSCs, the frequency of the cell surface markers was evaluated by flow cytometry. The data showed that WJ-MSCs expressed a high level of CD90 (94.5\%), CD44 (96.2\%), and CD73 (97.5\%), and a moderate level of CD105 (58\%) and CD106 (35.7\%). In order to find any possible contamination among the isolated cells, they were exposed to CD144 and CD34 antibodies that detected endothelial and hematopoietic cell markers, respectively. A negligible percentage of the isolated cells could express CD34 (1.21\%) and CD144 (1.13\%). These cells showed the capability to differentiate into adipocytes and osteoblasts. Oil red O staining revealed the presence of lipid droplets in differentiated adipocytes. Alizarin red S staining showed the precipitation of calcium by newly formed osteoblasts. Besides, the oil red staining of naïve WJ-MSCs revealed that there was no adipocyte contamination.

\subsection{Scaffold Characterization}

The scaffolds were fabricated in a disk-form construct (Figure 1A) and stained with toluidine blue for confirming the presence of heparin. Heparin contains negative charges located close to each other, so that the toluidine blue molecules can polymerize and emit a longer wavelength. The heparin-containing sponges stained metachromatic while collagen sponges without heparin stained orthochromatophilic (Figure 1B).

The FTIR spectra were obtained from crosslinked collagen with or without heparin, non-crosslinked collagen, and heparin per se. In the non-crosslinked collagen spectrum, absorption of carboxyl and amine-free groups were clearly observed centered around $3300 \mathrm{~cm}^{-1}$. Also, symmetric stretching vibrations of the COO-groups were shown at $1403 \mathrm{~cm}^{-1}$. The absorptions of carboxyl and amine groups were widened after crosslinking. This phenomenon occurs due to the formation of hydrogen binding. In addition, the intensity of amide I peak near $1650 \mathrm{~cm}^{-1}$, namely $\mathrm{C}=$ O stretching vibrations, increased slightly after crosslinking. These alterations in the FTIR spectra of the samples indicate the successful crosslinking procedure. Looking at heparin and heparin-collagen spectra, carboxyl and amine bonds had lower intensities compared to collagen sample. Peaks around $3400 \mathrm{~cm}^{-1}$ in heparinized-collagen indicate $\mathrm{N}-\mathrm{H}$ and $\mathrm{O}-\mathrm{H}$ stretching vibrations. The increase in $\mathrm{N}-\mathrm{H}$ bending vibration close to $1540 \mathrm{~cm}^{-1}$ is called the amide II band (Figure $1 \mathrm{C}$ ).

The SEM micrographs showed that both heparinized and non-heparinized sponges contained interconnected pores and the presence of heparin did not change the porosity of sponges. The image J software analysis showed that the percentages of surface porosity were $63.66 \%$ $\pm 5.46 \%$ for heparinized and $66.83 \% \pm 3.66 \%$ for nonheparinized scaffolds, without any significant betweengroup difference. The mean value of the pore surface area was $1375 \pm 774.97 \mu \mathrm{m}^{2}$ for heparinized and $387.38 \pm 352.48$ 


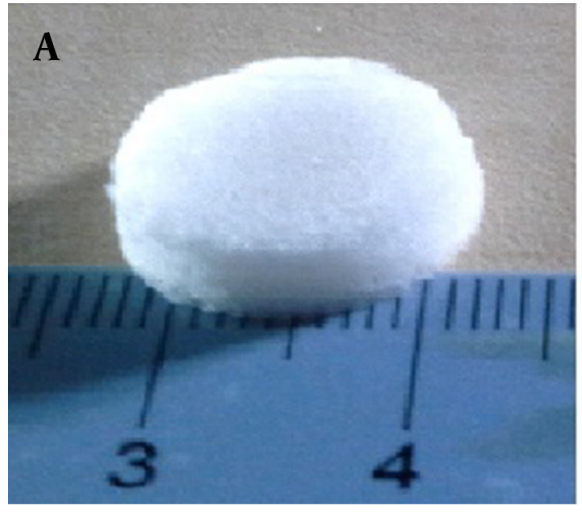

C

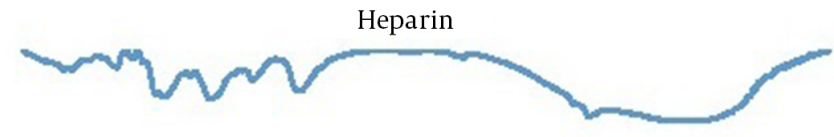

Crosslinked Collagen + Heparin

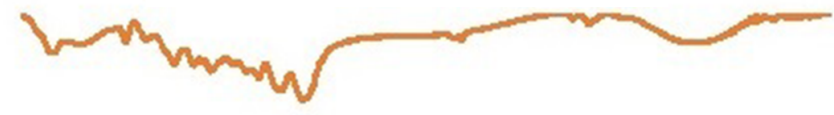

Crosslinked Collagen - Heparin
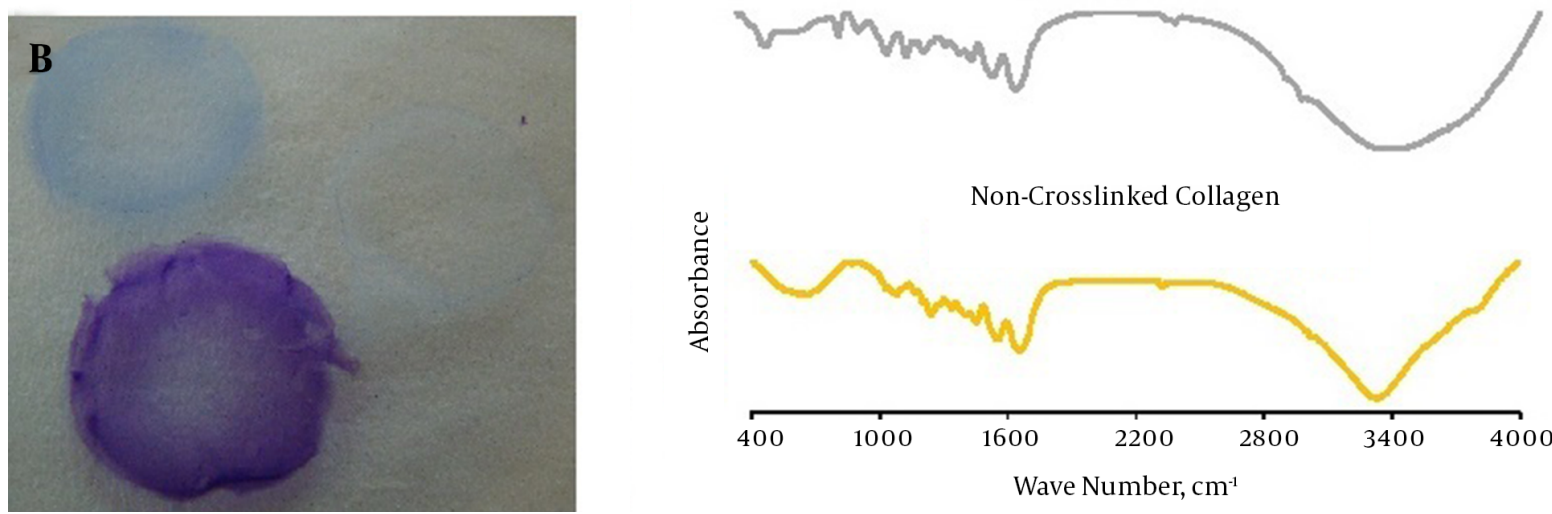

Figure 1. A sample of the fabricated sponge (A); toluidine blue staining, the purple color indicates the presence of heparin. Non-heparinized sponge stained blue (B); FIIR of heparin (blue), the crosslinked heparinized collagen sponge (orange), non-heparinized collagen sponges (gray) and non-crosslinked collagen sponges (yellow); the spectra of crosslinked heparinized collagen indicated that crosslinking had occurred (C).

$\mu \mathrm{m}^{2}$ for non-heparinized scaffolds. The coherence index showed randomly orientated fibers in both scaffolds. The orientation and coherency were $14.66^{\circ}$ and 0.118 in nonheparinized and $19.43^{\circ}$ and 0.061 in heparinized scaffolds, respectively (Figure $2 \mathrm{D}$ and $\mathrm{E}$ ).

\subsection{Cell Viability and Morphology}

The cell viability assay showed that heparin sponges had lower viability and metabolism compared with collagen non-heparinized sponge 3 days after culture. However, by extending the culture intervals, the metabolism of the cells increase in the presence of heparin. The best heparin concentration for long-term culture was $0.25 \mathrm{mg} / \mathrm{mL}$ which had a higher viability compared to all other groups, even after 14 days of culture. $1 \mathrm{mg} / \mathrm{mL}$ of heparin appeared to induce toxicities and therefore led to extensive cell death. In general, compared to the results obtained using collagen sponges, adding $0.25 \mathrm{mg} / \mathrm{mL}$ of heparin did not change the cell viability and metabolism considerably (Figure 3). With regard to these considerations, the sponges with $0.25 \mathrm{mg} / \mathrm{mL}$ heparin were selected for further assessments.

The cell morphology and distribution within the constructs were evaluated using SEM images. Hoechst and $\mathrm{H}$ and E staining of the cells seeded sponges. The cells with numerous processes were detected in SEM images (Figure 2A). Hoechst and H\&E staining indicated that most of the cells were concentrated close to the collagen fibers in both heparinized and non-heparinized sponges (Figure $2 \mathrm{~B}$ and C).

\subsection{Cell Differentiation Analysis}

Cells cultured in both heparinized and nonheparinized sponges in the presence of hepatogenic medium showed the expression of albumin, cytokeratin 18 and 19 and alpha-fetoprotein (Figure 4). Quantification of the reaction intensity by Image J showed a significantly higher intense staining for albumin, cytokeratin 19 and 18 and alpha-fetoprotein in the cells treated with hepatogenic medium and cultured within heparinized scaffolds ( $P=0.001,0.029,0.001$, and 0.001, respectively) (Figure 4). 


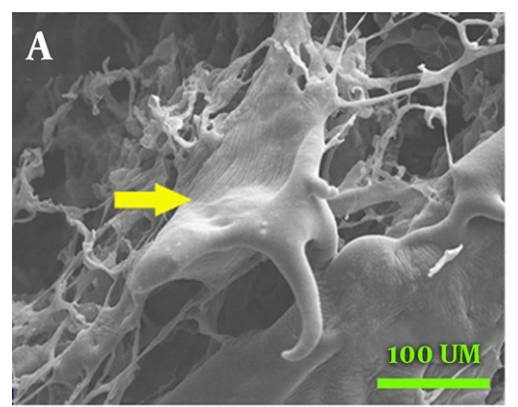

Without Heparin

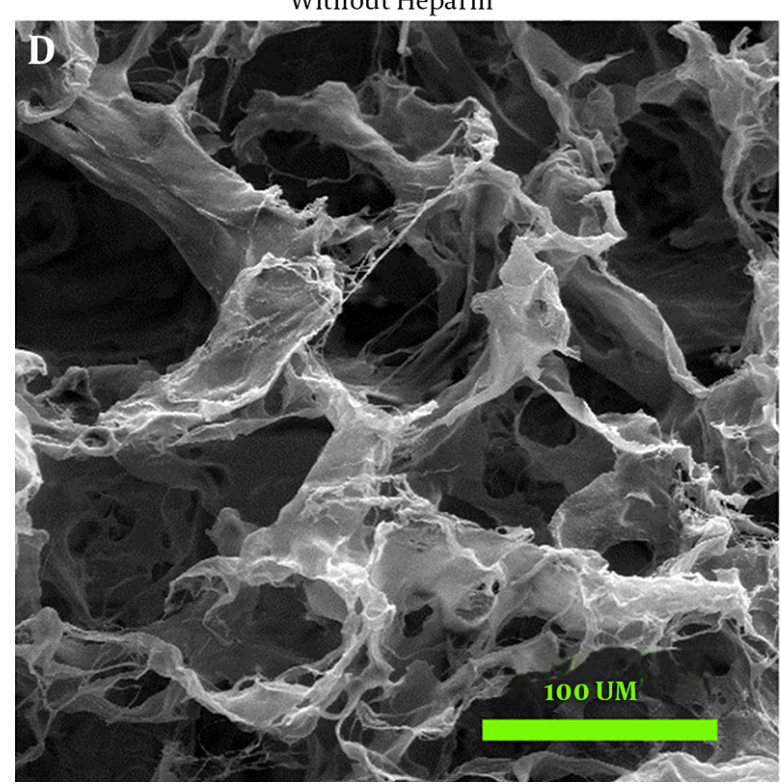

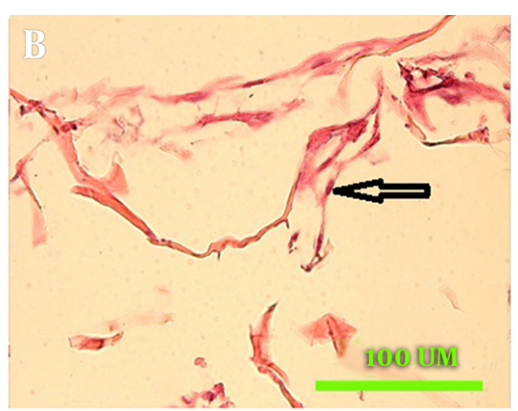

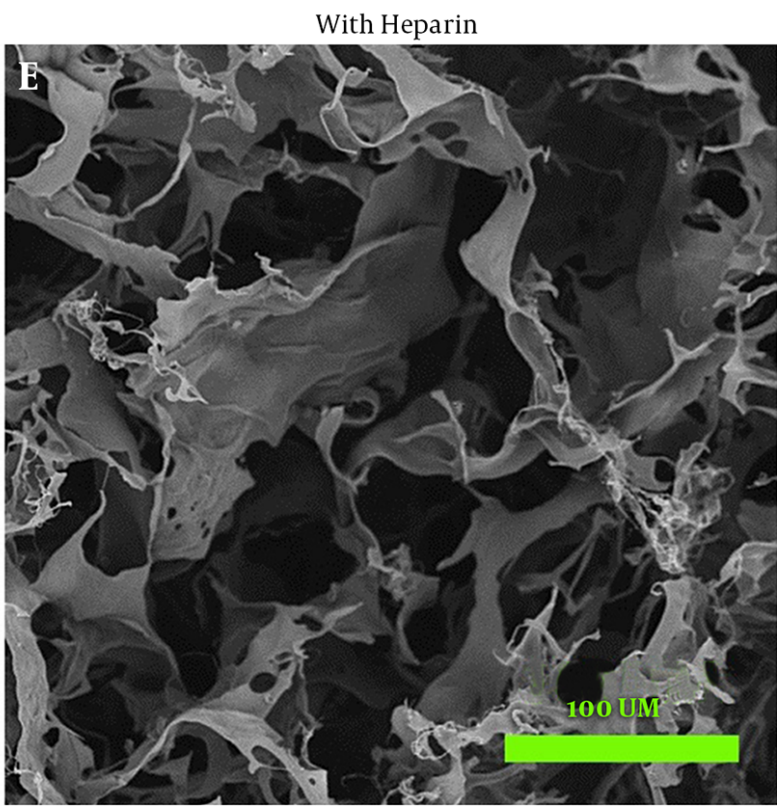

SEM image shows the cell (arrow) expand on the scaffold (A); light microscopy of the section stained with H\&E shows most of the cells (arrow) are located at the periphery of pores and attached to collagen fibers (B); hoechst stained cell nucleus (arrow) shows the distribution of the cells (C); scanning electron microscopy shows the porosity and fiber orientation of non-heparinized (D) and heparinized sponges (E).

The frequencies of the cells reacting with anti-cytokeratin 19 and albumin antibodies were significantly higher in cultures on the heparinized sponges $(\mathrm{P}=0.001$ and 0.018 , respectively). However, the statistical analysis revealed that the percentage of cytokeratin 18 and alpha-fetoprotein reacting cells did not change in the presence of heparin in the sponges (Figure 4). The data from indocyanine green analysis showed cell cultures on both heparinized and non-heparinized cultures could be functional and the cells on heparinized sponges showed non-significant higher releasing amounts of the dye. However, the level of uptake and release of the dye was significantly less than that of positive control and significantly higher than that of negative control (Figure 5).

PCR showed that the cells cultured within heparinized sponges expressed a higher level of albumin than those cultured within non- heparinized sponges.

\section{Discussion}

Heparin-based biomaterials have been used in surrogate organs including cartilage (26), vessels (27), heart valves (28), and liver (17). Heparin has angiogenic properties once used in 3D sponges in the presence of growth factor (29). This bioactive material also helps immobilize the growth factors, and thus improves cell viability in vitro (30). More specifically for primary hepatocytes, in vitro cellular attachment, morphology, and function are improved by culturing on heparin/collagen I-coated culture dishes (17). Albumin production by primary hepatocytes has been reported to increase in the presence of 


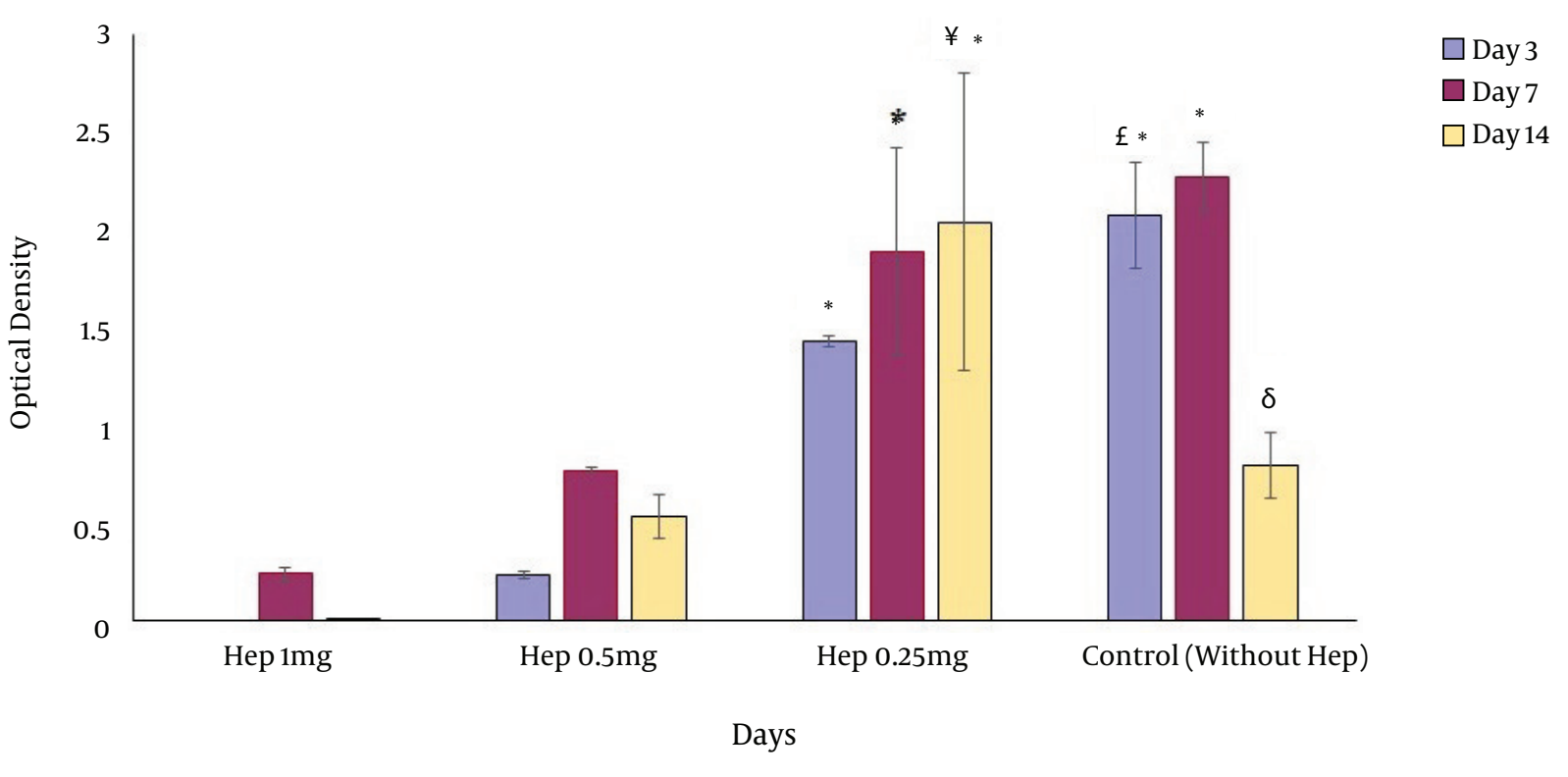

on the day 3, more viable cells were detected on 3D cultures containing $0.25 \mathrm{mg} / \mathrm{mL}$ of heparin and non-heparinized culture conditions. In long term culture, heparinized cultures except those contained $1 \mathrm{mg} / \mathrm{mL}$ showed a better condition for cell viability. *Significant difference with 3,7 and 14 days of cultures containing 1 and $0.5 \mathrm{mg} / \mathrm{mL}$ heparin $(\mathrm{P}<0.05), £$ Significant difference with 3 days of cultures containing $0.25 \mathrm{mg} / \mathrm{mL}$ heparin $(\mathrm{P}<0.05), \delta$ Significant difference with 14 days of cultures containing $0.25 \mathrm{mg} / \mathrm{mL}$ heparin $(\mathrm{P}<0.05)$, $¥$ Significant difference with 3,7 and 14 days of cultures containing 1 and $0.5 \mathrm{mg} / \mathrm{mL}$ heparin $(\mathrm{P}<0.05)$.

heparin (17). On the other hand, negative charges of heparin molecules have been suggested to inhibit cell proliferation (31). Therefore, here we first tried to find the right concentration of heparin that enhances the viability and metabolism of cells rather than inhibit their growth. Our data demonstrated an increase in cell viability, proliferation, and metabolism when WJ-MSCs were cultured in collagen scaffolds containing $0.25 \mathrm{mg} / \mathrm{mL}$ of heparin after 14 days compared to collagen non-heparinized 3D culture condition. However, the cell viability on heparinized ( 0.25 $\mathrm{mg} / \mathrm{mL}$ ) or non-heparinized sponges did not show a significant change compared to the control cultures after 3 days. Therefore, heparin, in $0.25 \mathrm{mg} / \mathrm{mL}$ heparinized collagen sponge, had not any short-term promotive effect once used more than 7 days in culture. Literature also showed the cell growth inhibitory effect of immobilized heparin on electrospun polycaprolacton/gelatin scaffold (32). Besides, short-term cultures revealed 3D culture system had a beneficial effect on cell viability regardless of the presence of heparin.

Heparin has been previously shown to influence the MSC proliferation, phenotype, and biological properties (33). Modification in biological properties may affect the differentiation capability of MSCs. The current study showed that culturing MSCs in heparinized 3D scaffold at the presence of hepatogenic media led to an increase in the frequency of albumin-and cytokeratin 19-positive cells and also a rise in the intensity of the reaction for all tested liverspecific markers. Heparinized collagen sponges have been detected to improve hepatocyte functions (20). Indocyanine green clearance test showed MSC-derived hepatocyte functions also are promoted in heparin/collagen sponges, as well.

Biomimicry improves the hepatocyte function in the organoid liver tissue. Heparin is one of the contents of Disse space and heparinized biomaterials imitate in vivo hepatocytes niche (34). Heparinized biomaterials have been shown to conduct primary hepatocyte phenotype and functional preservation (35). Heparin immobilizes the growth factors and modulates the cell access to the growth factors such as HGF (16). Coating of culture plates with crosslinked collagen IV/heparin has been reported to improve the hepatocyte differentiation capacity of bonemarrow-derived MSCs (36). Heparin treatment of decellularized scaffolds also improved the injected hepatocyte cell line, HepG2, and functionality after recellularization (37). The data showed that heparinized sponges could improve the WJ-MSC differentiation efficacy toward hepatocytes with higher functionality.

Glycosaminoglycans have been shown to increase 

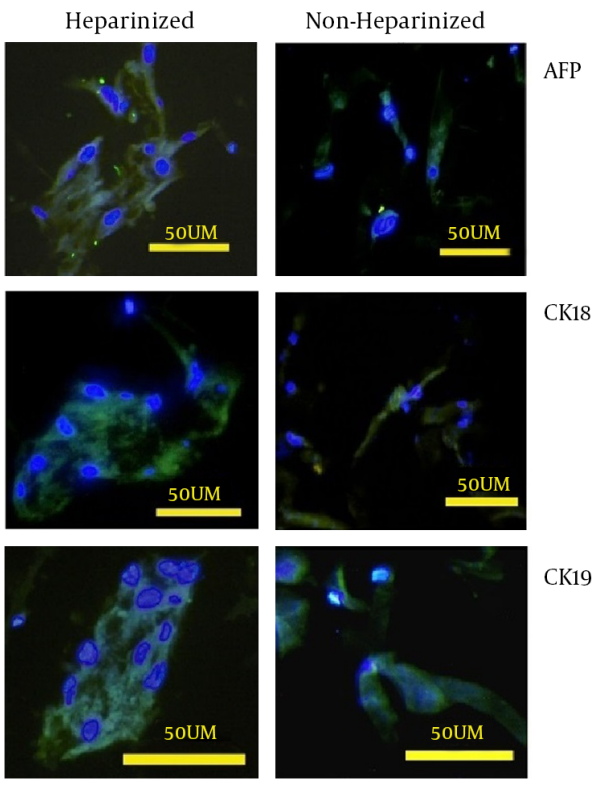

CK18
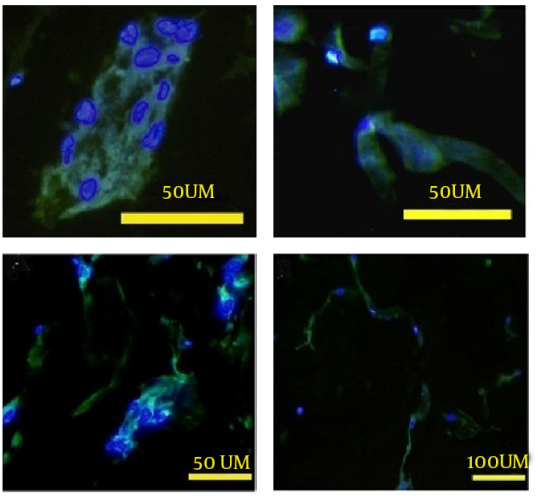

CK19
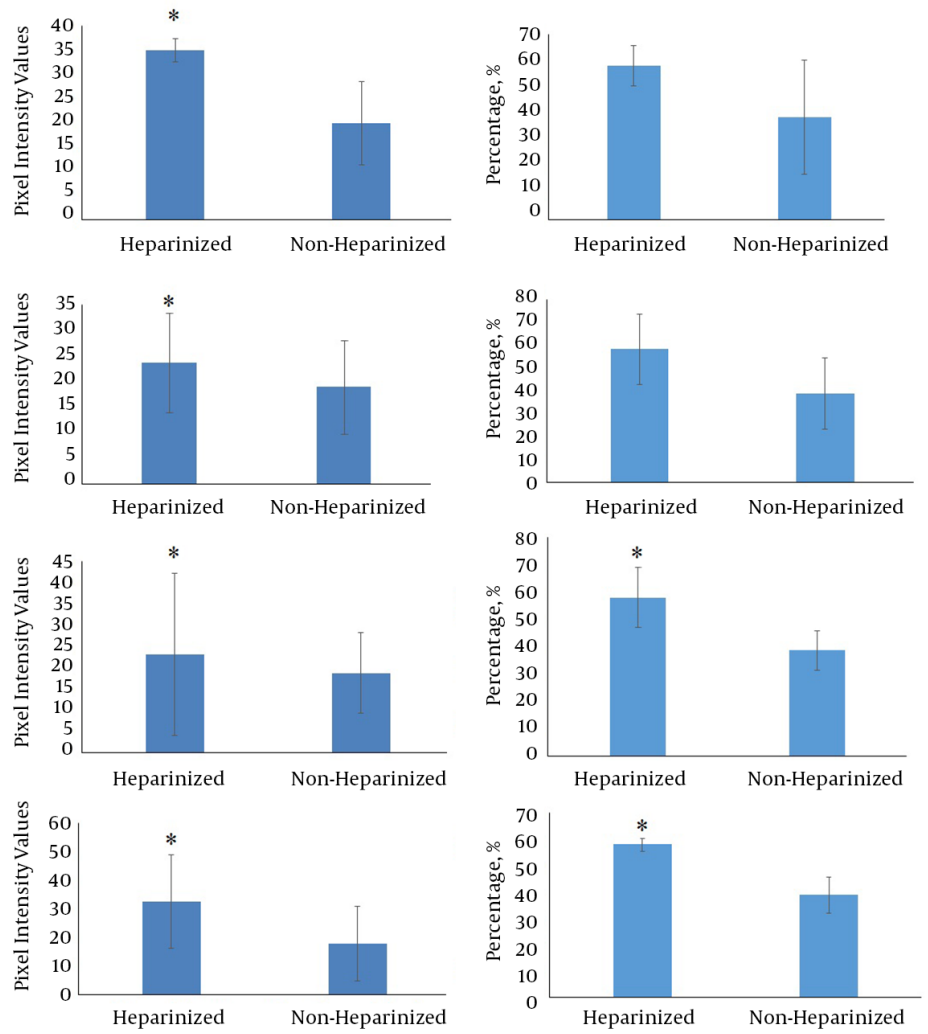

* Significant Difference with Non-Heparinized (P<O.O5)

The graphs at the middle are to compare the intensity of the cell reactions with the antibodies. The cells cultured in heparinized/collagen sponges stained more intense compared to non-heparinized/collagen sponges. The graphs at the right show the frequencies of positive cells to antibodies.

the endothelial outgrowth by interacting with heparinbinding growth factors (38). This provides a great opportunity for heparinized/collagen-3D sponges to be served as a good vehicle for cell therapy as well as for promoting angiogenesis. Heparin incorporated in gelatin scaffold has resulted in the enhanced endothelial cells migration (39). SEM images showed that both heparinized and non-heparinized sponges had an optimal porosity for cell growth; however, the mean value of the pore size of heparinized sponges was more than that in non-heparinized ones which might consequently protect better angiogenesis after transplantation and better nutrient and waste product exchange for in vitro hepatocyte toxicological and pharmacological experiments. The average pore size with a diameter of $40-70 \mu \mathrm{m}$ has been suggested to be appropriate for hepatocyte migration into the sponges and their functionality (40). Cell attachment and reduction in cell aggregation have been found to be affected by the pore size of the scaffolds (39). Our results showed the mean values of the pore size in the heparinized sponges were more than that of non-heparinized one. Porous integrity was demonstrated to be preserved by GAG (41). This range of the pore size is appropriate for hepatogenesis.

The research showed that physiochemical features of collagen I/ heparin sulfate sponges were appropriate for the cartilage (42). We also found that it is a good means for hepatocyte differentiation.

The hepatocytes lose their functions and phenotype when they are isolated from the natural niche $(8,9)$. Many in vitro models have been introduced to improve the hepatocyte activities and morphology (43). Such a model tries to imitate the in vivo niche complexities (44). The collagen/heparin scaffold can be introduced to be used in drug discovery and toxicological assessments as the data 
Figure 5. Indocyanine Green Uptake and Release Indicates the Functionality of the Differentiated Hepatocytes

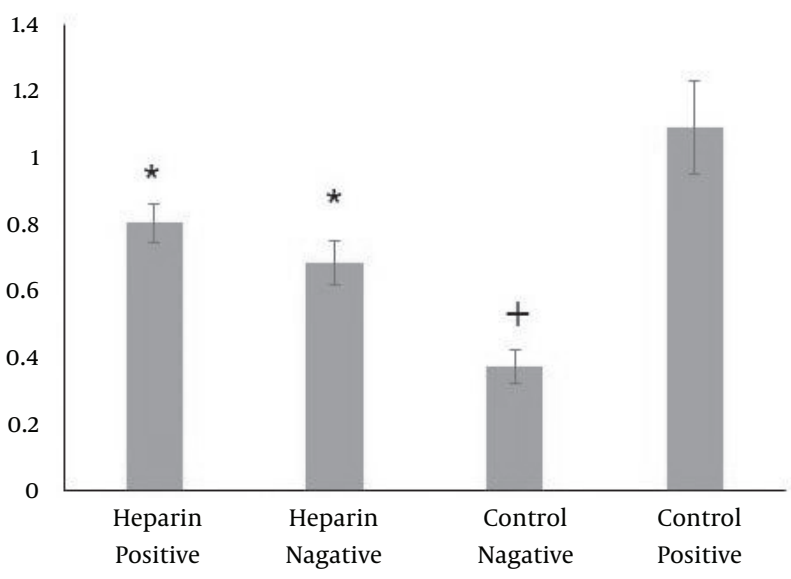

The cells differentiated into hepatocytes on the heparinized/collagen sponges. W] MSCs were used as negative control and HepG2 cell line was used as positive control. Eliminated more dye molecules compared to those cultured in nonheparinized/collagen sponges. ${ }^{*}$ Significant difference with the control groups ( $\mathrm{P}$ $<0.05) ;+$ significant difference with positive control $(\mathrm{P}<0.05)$

showed that it improved the stem cell-derived hepatocyte functionality.

\subsection{Conclusion}

Since heparin sequestrates the growth factors and protects them against degradation, its immobilization to biomaterials is beneficial for tissue engineering approaches. Besides, heparin provides an environment with closer similarities with natural hepatocyte niche. The stem cells differentiated into more functional hepatocytes in the heparinized scaffolds and therefore, it might be suggested as an appropriate engineered tissue for teratological researches, drug discovery, and use in regenerative medicine.

\section{Acknowledgments}

This work was supported by a grant provided by Iran national science foundation (grant \#91003812). We would like to thank M. Salmannejad and M. Roozitalab for technical support and Dr. N. Shokrpour at research improvement center of Shiraz University of Medical Sciences (SUMS) for improving English. We appreciate the anatomy department at SUMS for facilities provided to perform the experiments.

\section{Footnotes}

Authors' Contribution: Fatemeh Aleahmad: acquisition of data, analysis and interpretation of data, drafting of the manuscript, statistical analysis; Tahereh Talaei-Khozani: study concept and design, analysis and interpretation of data, critical revision of the manuscript for important intellectual content, statistical analysis, administrative, technical, and material support, fund acquisition, study supervision; Sareh Rajabi-Zeleti: technical support for scaffold fabrication, revision of manuscript; Mahsa Sani: technical support; Sasan Jalili-Firoozinezhad: technical support for scaffold fabrication; revision of manuscript; Shahin Bonakdar: FTIR technical support and interpretation; Sanaz Heshmat-Azad: FTIR interpretation ; Mahnaz Azarnia: study supervision; Mansoureh Jaberipour : study advisory.

Financial Disclosure: This project was supported by Iran national science foundation, INSF, the grant no 91003812

Role of the Sponsor: The funding organization is Iran national science foundation, INSF, that had a role as reviewer of the project draft.

\section{References}

1. Lopez AD, Mathers CD, Ezzati M, Jamison DT, Murray CJL, editors. Global Burden of Disease and Risk Factors. ; 2006.

2. Mathers CD, Loncar D. Projections of global mortality and burden of disease from 2002 to 2030. PLoS Med. 2006;3(11):442. doi: 10.1371/journal.pmed.0030442. [PubMed: 17132052].

3. Ige OO, Umoru LE, Aribo S. Natural products: A minefield of biomaterials. ISRN Materials Sci. 2012.

4. Michalopoulos GK, Bowen WC, Zajac VF, Beer-Stolz D, Watkins $S$, Kostrubsky V, et al. Morphogenetic events in mixed cultures of rat hepatocytes and nonparenchymal cells maintained in biological matrices in the presence of hepatocyte growth factor and epidermal growth factor. Hepatology. 1999;29(1):90-100. doi: 10.1002/hep.510290149. [PubMed: 9862855].

5. Dunn JC, Yarmush ML, Koebe HG, Tompkins RG. Hepatocyte function and extracellular matrix geometry: long-term culture in a sandwich configuration. FASEB J. 1989;3(2):174-7. [PubMed: 2914628].

6. Oe S, Fukunaka Y, Hirose T, Yamaoka Y, Tabata Y. A trial on regeneration therapy of rat liver cirrhosis by controlled release of hepatocyte growth factor. J Control Release. 2003;88(2):193-200. [PubMed: 12628327].

7. Bissell DM, Guzelian PS. Phenotypic stability of adult rat hepatocytes in primary monolayer culture*. Annals New York Academy Sci. 1980;349(1):85-98. doi: 10.1111/j.1749-6632.1980.tb29518.x.

8. Vanhaecke T, Rogiers V. Hepatocyte cultures in drug metabolism and toxicological research and testing. Methods Mol Biol. 2006;320:20927. doi: 10.1385/1-59259-998-2:209. [PubMed: 16719393].

9. McCarty WJ, Usta OB, Luitje M, Bale SS, Bhushan A, Hegde M, et al. A novel ultrathin collagen nanolayer assembly for 3-D microtissue engineering: Layer-by-layer collagen deposition for long-term stable microfluidic hepatocyte culture. Technology (Singap World Sci). 2014;2(1):67-74. doi: 10.1142/S2339547814500083. [PubMed: 24932459].

10. Nishida Y, Taniguchi A. A three-dimensional collagen-sponge-based culture system coated with simplified recombinant fibronectin improves the function of a hepatocyte cell line. In Vitro Cellular \& Developmental Biology-Animal. 2015:1-7.

11. Lee JS, Shin J, Park HM, Kim YG, Kim BG, Oh JW, et al. Liver extracellular matrix providing dual functions of two-dimensional substrate coating and three-dimensional injectable hydrogel platform 
for liver tissue engineering. Biomacromolecules. 2014;15(1):206-18. doi: 10.1021/bm4015039. [PubMed: 24350561].

12. Baharvand H, Hashemi SM, Kazemi Ashtiani S, Farrokhi A. Differentiation of human embryonic stem cells into hepatocytes in 2D and 3D culture systems in vitro. Int J Dev Biol. 2006;50(7):645-52. doi: 10.1387/ijdb.052072hb. [PubMed:16892178].

13. Vasavada VA, Praveen MR, Shah SK, Trivedi RH, Vasavada AR. Antiinflammatory effect of low-molecular-weight heparin in pediatric cataract surgery: a randomized clinical trial. American J Ophthalmol. 2012;154(2):252-8.

14. Sommer A, Rifkin DB. Interaction of heparin with human basic fibroblast growth factor: protection of the angiogenic protein from proteolytic degradation by a glycosaminoglycan. J Cell Physiol. 1989;138(1):215-20. doi: 10.1002/jcp.1041380129. [PubMed: 2910884].

15. Lyon M, Deakin JA, Rahmoune H, Fernig DG, Nakamura T, Gallagher JT. Hepatocyte growth factor/scatter factor binds with high affinity to dermatan sulfate. J Biol Chem. 1998;273(1):271-8. [PubMed: 9417075].

16. Kim M, Lee JY, Jones CN, Revzin A, Tae G. Heparin-based hydrogel as a matrix for encapsulation and cultivation of primary hepatocytes. Biomaterials. 2010;31(13):3596-603. doi: 10.1016/j.biomaterials.2010.01.068. [PubMed: 20153045].

17. You J, Raghunathan VK, Son KJ, Patel D, Haque A, Murphy CJ, et al. Impact of Nanotopography, Heparin Hydrogel Microstructures, and Encapsulated Fibroblasts on Phenotype of Primary Hepatocytes. ACS Appl Mater Interfaces. 2015;7(23):12299-308. doi: 10.1021/am504614e. [PubMed: 25247391].

18. Khodabandeh Z, Vojdani Z, Talaei-Khozani T, Jaberipour M, Hosseini A, Bahmanpour S. Comparison of the Expression of Hepatic Genes by Human Wharton's Jelly Mesenchymal Stem Cells Cultured in 2D and 3D Collagen Culture Systems. Iran J Med Sci. 2016;41(1):28-36. [PubMed: 26722142]

19. Wang J, Chen F, Liu L, Qi C, Wang B, Yan X, et al. Engineering EMT using 3D micro-scaffold to promote hepatic functions for drug hepatotoxicity evaluation. Biomaterials. 2016;91:11-22. doi: 10.1016/j.biomaterials.2016.03.001. [PubMed: 26994875].

20. Higuchi Y, Kawai K, Kanaki T, Yamazaki H, Chesne C, GuguenGuillouzo C, et al. Functional polymer-dependent 3D culture accelerates the differentiation of HepaRG cells into mature hepatocytes. Hepatol Res. 2016;46(10):1045-57. doi: 10.1111/hepr.12644. [PubMed: 26724677].

21. Rajan N, Habermehl J, Cote MF, Doillon CJ, Mantovani D. Preparation of ready-to-use, storable and reconstituted type I collagen from rat tail tendon for tissue engineering applications. Nat Protoc. 2006;1(6):2753-8. doi: 10.1038/nprot.2006.430. [PubMed:17406532].

22. Rajabi-Zeleti S, Jalili-Firoozinezhad S, Azarnia M, Khayyatan F, Vahdat S, Nikeghbalian S, et al. The behavior of cardiac progenitor cells on macroporous pericardium-derived scaffolds. Biomaterials. 2014;35(3):970-82. doi: 10.1016/j.biomaterials.2013.10.045. [PubMed: 24183165].

23. Kurpinski KT, Stephenson JT, Janairo RR, Lee H, Li S. The effect of fiber alignment and heparin coating on cell infiltration into nanofibrous PLLA scaffolds. Biomaterials. 2010;31(13):3536-42. doi: 10.1016/j.biomaterials.2010.01.062. [PubMed: 20122725].

24. Petsa A, Gargani S, Felesakis A, Grigoriadis N, Grigoriadis I. Effectiveness of protocol for the isolation of Wharton's Jelly stem cells in largescale applications. In Vitro Cell Dev Biol Anim. 2009;45(10):573-6. doi: 10.1007/s11626-009-9227-0. [PubMed:19609625].

25. Ho CM, Dhawan A, Hughes RD, Lehec SC, Puppi J, Philippeos C, et al. Use of indocyanine green for functional assessment of human hepatocytes for transplantation. Asian J Surg. 2012;35(1):9-15. doi: 10.1016/j.asjsur.2012.04.017. [PubMed: 22726558].

26. Recha-Sancho L, Semino CE. Heparin-based self-assembling peptide scaffold reestablish chondrogenic phenotype of expanded de-differentiated human chondrocytes. I Biomed Mater Res A. 2016;104(7):1694-706. doi:10.1002/jbm.a.35699. [PubMed: 26939919].

27. Ye L, Wu X, Duan HY, Geng X, Chen B, Gu YQ, et al. The in vitro and in vivo biocompatibility evaluation of heparin-poly(epsiloncaprolactone) conjugate for vascular tissue engineering scaffolds. J Biomed Mater Res A. 2012;100(12):3251-8. doi: 10.1002/jbm.a.34270. [PubMed: 22733560].

28. Flanagan TC, Wilkins B, Black A, Jockenhoevel S, Smith TJ, Pandit AS A collagen-glycosaminoglycan co-culture model for heart valve tissue engineering applications. Biomaterials. 2006;27(10):2233-46. doi: 10.1016/j.biomaterials.2005.10.031. [PubMed: 16313955].

29. Nillesen ST, Geutjes PJ, Wismans R, Schalkwijk J, Daamen WF, van Kuppevelt $\mathrm{TH}$. Increased angiogenesis and blood vessel maturation in acellular collagen-heparin scaffolds containing both FGF2 and VEGF. Biomaterials. 2007;28(6):1123-31. doi: 10.1016/j.biomaterials.2006.10.029. [PubMed: 17113636].

30. Hou YT, Ijima H, Takei T, Kawakami K. Growth factor/heparin immobilized collagen gel system enhances viability of transplanted hepatocytes and induces angiogenesis. JBiosci Bioeng. 2011;112(3):26572. doi: 10.1016/j.jbiosc.2011.05.003. [PubMed: 21640648].

31. Nikitovic D, Zafiropoulos A, Tzanakakis GN, Karamanos NK, Tsatsakis AM. Effects of glycosaminoglycans on cell proliferation of normal osteoblasts and human osteosarcoma cells depend on their type and fine chemical compositions. Anticancer Res. 2005;25(4):2851-6. [PubMed: 16080537].

32. Lee J, Yoo JJ, Atala A, Lee SJ. The effect of controlled release of PDGF-BB from heparin-conjugated electrospun PCL/gelatin scaffolds on cellular bioactivity and infiltration. Biomaterials. 2012;33(28):6709-20. doi 10.1016/j.biomaterials.2012.06.017. [PubMed: 22770570].

33. Ling L, Camilleri ET, Helledie T, Samsonraj RM, Titmarsh DM, Chua RJ et al. Effect of heparin on the biological properties and molecular signature of human mesenchymal stem cells. Gene. 2016;576(1 Pt 2):292303. doi: 10.1016/j.gene.2015.10.039. [PubMed: 26484394].

34. Dudas J, Bocsi J, Fullar A, Baghy K, Fule T, Kudaibergenova S. Heparin and liver heparan sulfate can rescue hepatoma cells from topotecan action. Biomed Res Inter. 2014.

35. You J, Shin DS, Patel D, Gao Y, Revzin A. Multilayered heparin hydrogel microwells for cultivation of primary hepatocytes. Adv Healthc Mater 2014;3(1):126-32. doi: 10.1002/adhm.201300054. [PubMed: 23828859].

36. Razban V, Khajeh S, Lotfi AS, Mohsenifar A, Soleimani M, Khoshdel A, et al. Engineered heparan sulfate-collagen iv surfaces improve human mesenchymal stem cells differentiation to functional hepatocyte-like cells. J Biomaterials Tissue Engin. 2014;4(10):811-22. doi: 10.1166/jbt.2014.1234.

37. Hussein KH, Park KM, Kang KS, Woo HM. Heparin-gelatin mixture improves vascular reconstruction efficiency and hepatic function in bioengineered livers. Acta Biomater. 2016;38:82-93. doi: 10.1016/j.actbio.2016.04.042. [PubMed: 27134015].

38. Chevalier F, Lavergne M, Negroni E, Ferratge S, Carpentier G, GilbertSirieix $M$, et al. Glycosaminoglycan mimetic improves enrichment and cell functions of human endothelial progenitor cell colonies. Stem Cell Res. 2014;12(3):703-15. doi:10.1016/j.scr.2014.03.001. [PubMed: 24681520].

39. Leijon J, Carlsson F, Brannstrom J, Sanchez J, Larsson R, Nilsson B, et al Attachment of flexible heparin chains to gelatin scaffolds improves endothelial cell infiltration. Tissue Eng Part A. 2013;19(11-12):1336-48. doi: 10.1089/ten.TEA.2011.0712. [PubMed: 23327585].

40. Tripathi A, Melo JS. Preparation of a sponge-like biocomposite agarose-chitosan scaffold with primary hepatocytes for establishing an in vitro 3D liver tissue model. RSC Adv. 2015;5(39):30701-10. doi: 10.1039/c5ra04153h.

41. Pieper JS, Hafmans T, Veerkamp JH, van Kuppevelt TH. Development of tailor-made collagen-glycosaminoglycan matrices: EDC/NHS crosslinking, and ultrastructural aspects. Biomaterials. 2000;21(6):581-93. [PubMed: 10701459].

42. Sanjurjo-Rodriguez C, Martinez-Sanchez AH, Hermida-Gomez T, Fuentes-Boquete I, Diaz-Prado S, Blanco FJ. Differentiation of human mesenchymal stromal cells cultured on collagen sponges 
for cartilage repair. Histol Histopathol. 2016;31(11):1221-39. doi: 10.14670/HH-11-754. [PubMed: 26965505].

43. Dash A, Inman W, Hoffmaster K, Sevidal S, Kelly J, Obach RS, et al. Liver tissue engineering in the evaluation of drug safety. Expert Opin Drug Metab Toxicol. 2009;5(10):1159-74. doi: 10.1517/17425250903160664.
[PubMed: 19637986].

44. Lin C, Ballinger KR, Khetani SR. The application of engineered liver tissues for novel drug discovery. Expert Opin Drug Discov. 2015;10(5):51940. doi: 10.1517/17460441.2015.1032241. [PubMed: 25840592]. 